\title{
Embedded acoustic emission system based on rock sound source crowd location
}

\author{
Zhang Qing
}

\begin{abstract}
Rock acoustic emission is often used to study the evolution of brittle materials. The cause of rock internal damage can be monitored continuously and real-timely by sensing rock acoustic wave. However, the key problem is how to split the position and analyze the acoustic emission characteristics based on the acoustic tracking experiment. First, we collected the acoustic waves of rock evolution. Then, a linear relationship is established between the acoustic emission and the propagation process of rock. And we proposed a rock sound source crowd localization algorithm. The algorithm can accurately restore the internal deformation and failure process of the rock. Finally, we designed an acoustic emission characteristic analysis method based on the acoustic source and rock sound. Test results of the ten location algorithm show that the positioning error of the proposed algorithm is less than $0.005 \mathrm{~m}$, which is obviously better than the least square method. The calculation of rock strength and splitting rate is consistent with the actual measurement by using the embedded acoustic emission characteristic analysis mechanism.
\end{abstract}

Keywords: Acoustic emission, Embedded systems, Rock sound source, Crowd location

\section{Introduction}

In the process of rock breaking, different sound waves are emitted [1]. Through the analysis of rock acoustic emission characteristics, the spatial and temporal evolution process of rock can be established [2,3]. It is of great significance to study the [4] evolution and prediction of rock fracture.

About the research of acoustic emission system, there are the following articles. Cai, $\mathrm{Z}$ et al. studied the mechanism of electromagnetically induced acoustic emission based on the electrical activated plastic concept and mobile dislocation model [5], a new approach for unsupervised pattern recognition in acoustic emission time-series issued from composite materials in the article [6]. In the article [7], Ming A B et al. used the acoustic emission signal to evaluate the size of a single spall by considering the sensitivity of the signal to the incipient fault detection.

About the sound localization achievements were as follows. The survey of the state of the art in source location privacy was provided in article [8]. The path extension method of article [9] could provide strong

Correspondence: cnzhangqingcn@sina.com

School of Civil Engineering, Jilin Jianzhu University, Changchun 130118, China protection for source-location privacy, which performs quite well even though an object occurs near the base station. The method proposed by Tang Y et al. [10] has a solid theoretical basis, which can locate voltage sag source with an accuracy of $100 \%$, in power networks with various topologies.

The research and deployment of acoustic wave acquisition equipment are ignored in the above research. For the analysis of rock acoustic emission activity characteristics, the real-time performance is still not satisfied. We designed a kind of rock sound source crowd localization algorithm. Then, the acoustic emission characteristic analysis method is studied, which is embedded in the rock.

The rest of the paper is organized as follows. Section 2 describes the rock sound source crowd localization algorithm. In Section 3, we design the embedded acoustic emission characteristic analysis mechanism. Performance evaluations are given in Section 4. Finally, we conclude the paper in Section 5.

\section{Rock sound source crowd localization algorithm}

The deformation and cracking of the rock will transmit sound waves. The evolution of rocks and the expansion of the companion will transmit sound waves. The rock acoustic emission and propagation process has a linear

\section{Springer Open}

(c) 2016 The Author(s). Open Access This article is distributed under the terms of the Creative Commons Attribution 4.0 International License (http://creativecommons.org/licenses/by/4.0/), which permits unrestricted use, distribution, and reproduction in any medium, provided you give appropriate credit to the original author(s) and the source, provide a link to the Creative Commons license, and indicate if changes were made. 
relationship with the change of rock. By analyzing the emission source and signal strength of rock sound, the driving factors of rock structure can be obtained. The evolution degree of rock structure can be obtained by analyzing the signal strength of rock sound. Therefore, it is the key to realize the localization and emission monitoring of rock students. The evolution of rock structure will lead to the imbalance of the internal energy of the rock. Constructing the structure of the internal imbalance of rock is by monitoring the sound of rock.

Three rock groups of different structures are shown in Fig. 1; a block of rock, a cylinder of rock, a piece of stone. The evolution of rock structure caused the interaction of the three rocks with each other. Parameter $Y$ indicates the direction of the rectangular rock force. The parameter $x$ indicates the direction of the force of the cylinder. $\alpha$ is the angle between the acoustic emission direction of the rectangular rock and the trapezoidal rock. $\beta$ indicates the angle between the acoustic emission direction of the cylinder rock and the trapezoid rock.

The acoustic wave force field in the evolution process of rock group is shown in formula (1).

$$
\left\{\begin{array}{l}
\delta_{x} \\
\delta_{y} \\
\sqrt{\delta_{x}+\delta_{y}}
\end{array}\right\}=\frac{\cos \alpha}{\pi \sqrt{\sin \beta}}\left\{\begin{array}{l}
1-y \sin \alpha \sqrt{\left|x^{2}-y^{2}\right|} \\
1+y \cos \alpha \sqrt{\left|x^{2}-y^{2}\right|} \\
y \cos \frac{\beta}{2} \sin \alpha \sqrt{\left|x^{2}-y^{2}\right|}
\end{array}\right\}
$$

Here, $\delta_{x}$ denotes the rock $s$ sound wave stress field from the cuboid cylindrical rock. $\delta_{y}$ denotes the rectangular rock acoustic force field from cylinder rock and trapezoidal rock.

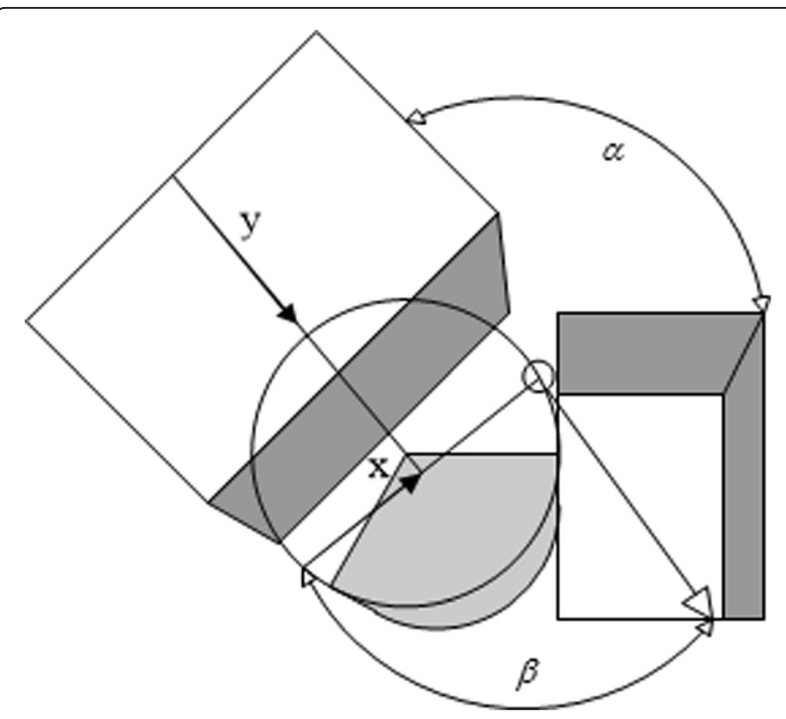

Fig. 1 Heterogeneous rock group
Internal energy evolution of rock group is shown in formula (2).

$$
\left\{\begin{array}{l}
\eta_{1} \\
\eta_{2} \\
\eta_{3}
\end{array}\right\}=\frac{\sqrt{\left|x^{2}-y^{2}\right|}}{\pi \sqrt{\cos \beta}}\left\{\begin{array}{c}
\cos \left(\alpha^{2}-\beta^{2}\right) \mu \\
|x-y|^{\mu} \cos \alpha \\
\mu \sin \frac{\alpha}{2} \cos \frac{\beta}{2}
\end{array}\right\}
$$

Here, the energy intensity of the rectangular rock is expressed by $\eta_{1}$. Energy field strength of cylinder rocks is denoted by $\eta_{2}$. Energy intensity of the trapezoidal rock is denoted by $\eta_{3}$. Energy loss weight is denoted by $\mu$.

The structure of the rock group deformation and acoustic propagation direction is shown in Fig. 2. Here, $\gamma$ is the angle between weakening of energy and acoustic emission in evolution of the cylinder rock. The angle between the acoustic propagation direction of structure deformation and the direction of energy weakening in the direction of sound propagation and the direction of energy weakening is $w$. Therefore, the displacement caused by the structural deformation of the rock group is shown in the formula (3).

$$
\left\{\begin{array}{l}
\Delta x \\
\Delta y
\end{array}\right\}=\frac{\mu}{\sqrt{2 \pi}}\left\{\begin{array}{l}
\frac{1}{2} \cos \alpha \cos \gamma \\
\frac{1}{2} \sin \omega \cos \frac{1}{2} \beta
\end{array}\right\}
$$

The moving distance of rock group structure deformation $\sqrt{\Delta x^{2}+\Delta y^{2}}$ can be obtained. The rock acoustic energy $A_{U}$ could be obtained by formula (4).

$$
A_{U}=\left\{\begin{array}{l}
\frac{2}{\mu}\left|\sqrt{\Delta x^{2}+\Delta y^{2}}-\delta_{x} \delta_{y}\right| \sin \omega, \sqrt{\Delta x^{2}+\Delta y^{2}}>\sqrt{\left|x^{2}-y^{2}\right|} \\
\frac{\pi}{2} \sqrt{\left|x^{2}-y^{2}\right|} \cos \gamma, \sqrt{\Delta x^{2}+\Delta y^{2}} \leq \sqrt{\left|x^{2}-y^{2}\right|}
\end{array}\right.
$$

In order to better monitor the acoustic emission of rock or large rock groups, we designed a wireless rock sound source monitoring network based on swarm intelligence. The network has the following functions:

(1)Monitoring and convergence of rock acoustic emission signals

(2) The reason and classification of rock acoustic emission

(3) The structure and location of the rock sound source

(4) The location of the rock or rock group

Based on the rock mechanical field, acoustic emission and energy field, the three-dimensional space of the sound source localization sensor based on the group wise perception is established, which is shown in Fig. 3. Here, $Z_{1}, Z_{2}, Z_{3}, Z_{4}$ are the rock acoustic 


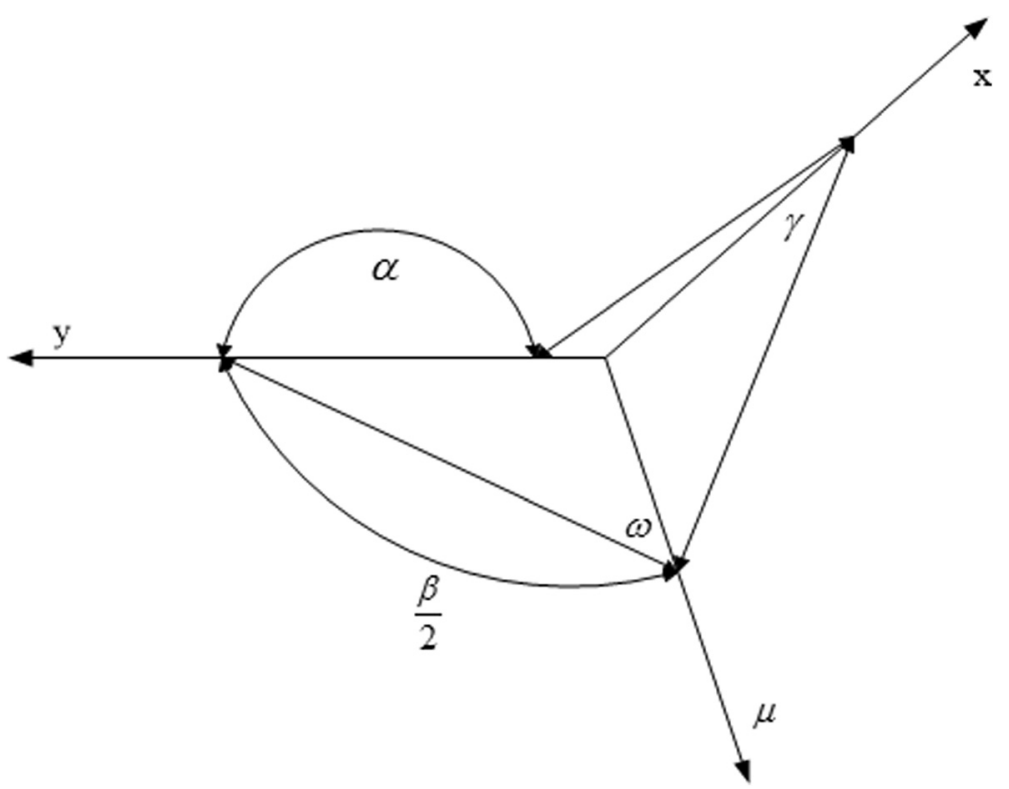

Fig. 2 Structural deformation and propagation of rock groups

emission signals perceived by the swarm intelligence sensors, which belong to the reverse direction of $Y$ in the three bit space. In order to facilitate the sound source localization, they are mapped to the $Y$ forward. In Fig. 3, we found that there are two perceptual points $Z_{2}^{\prime}$ on the positive and the positive direction of the $X$ and $Z_{\mu}$. This two-point is mapped to $Z_{2}$ point. This is because the characteristic of the formula (5) is shown when the rock acoustic emission is perceived by the swarm intelligence sensor.

$$
Z_{2}=\left\{\begin{array}{l}
\frac{1}{2} \cos \alpha \sin \omega, \Delta x>x_{2} \\
\frac{1}{2 \pi} \sin \beta \operatorname{cso} \gamma, \mu_{2}>\Delta \mu
\end{array}\right.
$$

Rock sound source crowd positioning algorithm workflow is shown below:

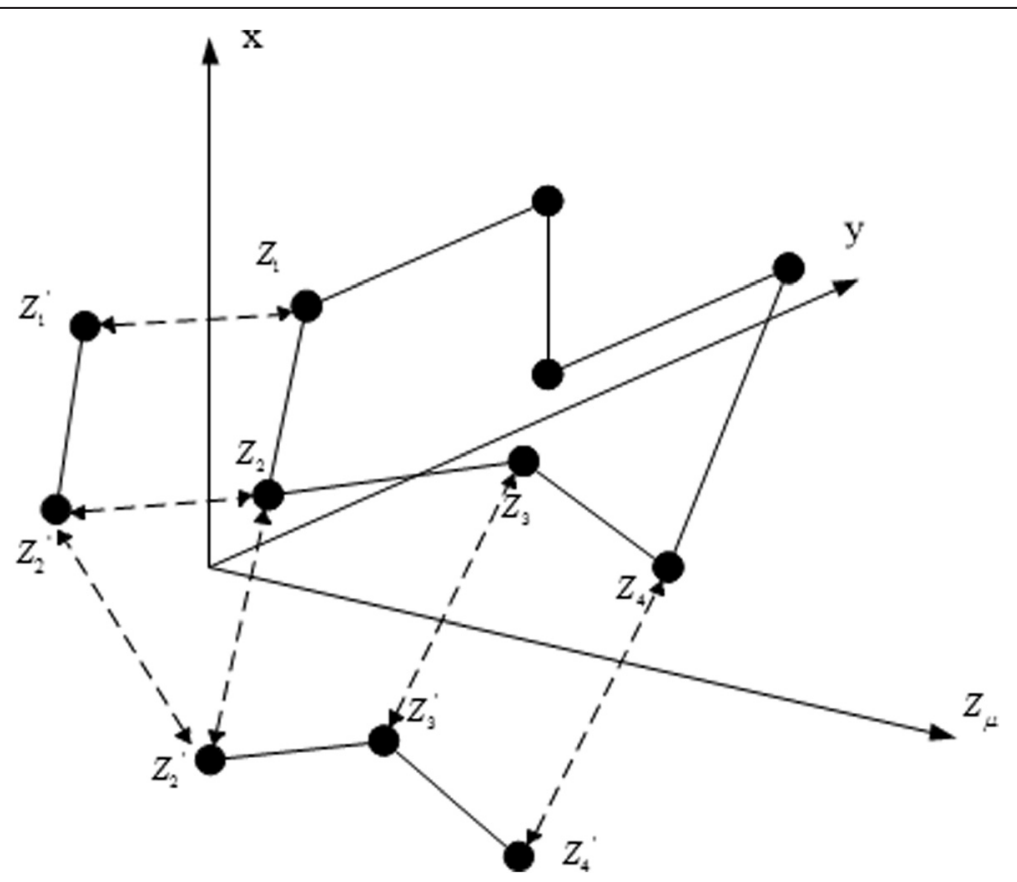

Fig. 3 Three dimensional space of sound source localization sensor 
(1)Monitoring the acoustic emission energy of each rock group

(2)Determination of rock structure

(3) Calculate the displacement vector of rock structure

(4) Crowd sensors to perceive the three force and harmonic propagation process of rock group

(5)Mapping all the swarm intelligence to forward space of $y$

(6) To connect the $y$ to the space of the crowd perception points to 1 closed loop, to achieve the sound source localization

\section{Embedded acoustic emission characteristic analysis mechanism}

After receiving the external force, rock would produce deformation. Rock structure may be splitting. The internal energy would be released in the splitting process. Through the crowd positioning sound source in Section 2, the internal deformation and failure process of rock can be reduced by the combination of sound source and rock sound. We design a method to analyze the acoustic emission characteristics of the swarm intelligence sensors embedded in rock. The analysis model of internal acoustic emission energy of rock or rock group is established to provide a basis for the study of the evolution process of rock.

Taking the cylinder rock as the object, the embedding group intelligence sensor node is shown in Fig. 4, the acoustic emission range of the rock after splitting. We embedded five groups of sensor nodes. Among them, 1, 3 , and 4 nodes are embedded in the main part of the rock. The node 3 is completely embedded in the separation of the rocks. The node 5 is embedded in the separation of the rock and the intersection of the main body.

According to the formula (6), the 3D space acoustic emission sample series of the $x, y$, and $Z_{\mu}$ are embedded in fitting according to the formula (6). In the time dimension, the statistical $N$ embedded crowd sensor node monitoring to the rock sound, get the rock acoustic emission energy $E$. Then, the analysis of the displacement of the splitting of the rock evolution is obtained, and the coordinates of all the separated bodies $(x, y)$ are obtained. Based on the angle of acoustic emission and displacement distance, the evolution factor vector of internal structure of rock is obtained.

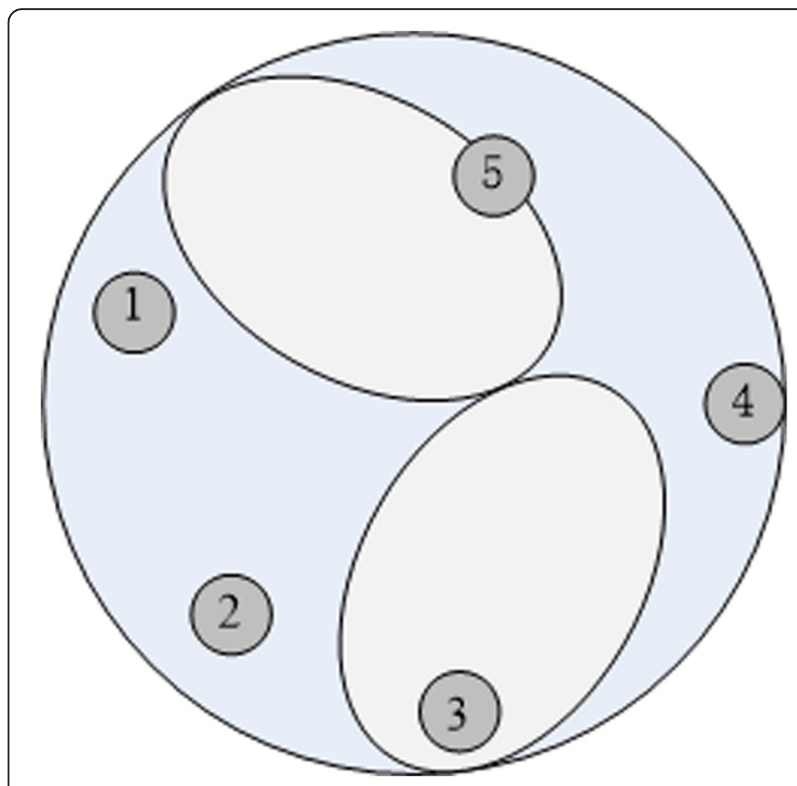

Fig. 4 Cylinder rocks embedded in a group of sensor nodes

$$
\left\{\begin{array}{l}
E(t)=\mu+\int_{i=1}^{N} A_{U}(t) d t \\
(x, y)=\iint_{t} \cos \left(w \lg \left(t-\sum_{i=1}^{N} t_{i}\right)\right) d x d y \\
Z_{\mu}=\frac{\tan |\alpha-\beta|}{\gamma}|\Delta x-\Delta y|
\end{array}\right.
$$

The acoustic emission characteristics of rock bodies shown in Fig. 4 must be satisfied with the embedded sensor node distribution characteristics and sampling parameters shown in Table 1 . In addition, in order to accurately analyze the characteristics of embedded acoustic emission, the splitting activities of rock bodies or rock groups should meet the activities of Table 2. Tables 1 and 2 are given the range of values, as the reference value of the embedded node in the rock. The parameter $M$ of Table 1 indicates the total sample number. The parameter $a$ represents the maximum height of the rock body. The parameter $b$ represents the maximum width of the rock body. El represents the total

Table 1 Distribution characteristics of embedded sensor nodes

\begin{tabular}{llllll}
\hline Node & Sample number & $x$ & $y$ & $Z_{\mu}$ & Location \\
\hline 1 & $13 \% \mathrm{M}$ & $0.3 \mathrm{a}+0.7 \mathrm{~b}$ & $0.1 \mathrm{a}+0.9 \mathrm{~b}$ & $0.3 \mathrm{El}$ & {$[a \cos a, b \sin \beta]$} \\
2 & $13 \% \mathrm{M}$ & $0.3 \mathrm{a}+0.7 \mathrm{~b}$ & $0.1 \mathrm{a}+0.9 \mathrm{~b}$ & $0.3 \mathrm{El}$ & {$[a \cos a, b \sin \beta]$} \\
3 & $25 \% \mathrm{M}$ & $0.4 \mathrm{a}+0.6 \mathrm{~b}$ & $0.8 \mathrm{a}+0.2 \mathrm{~b}$ & $0.38 \mathrm{El}$ & {$\left[a \cos \frac{a}{2}, b \sin \beta\right]$} \\
4 & $0.3 \mathrm{a}+0.7 \mathrm{~b}$ & $0.1 \mathrm{a}+0.9 \mathrm{~b}$ & $0.3 \mathrm{El}$ & {$[a \cos a, b \sin \beta]$} \\
5 & $13 \% \mathrm{M}$ & $0.6 \mathrm{a}+0.4 \mathrm{~b}$ & $0.5 \mathrm{a}+0.5 \mathrm{~b}$ & $0.6 \mathrm{El}$ & {$\left[a \cos a, b \sin \frac{\beta}{2}\right]$} \\
\hline
\end{tabular}


Table 2 Split activity curve

\begin{tabular}{lllll}
\hline Node & $a$ & $\beta$ & $\gamma$ & $\omega$ \\
\hline 1 & $|a-b|^{2} \phi$ & $\phi$ & $\mu \phi$ & $|\Delta x-\Delta y| \phi$ \\
2 & $|a-b|^{2} \phi$ & $\phi$ & $\mu \phi$ & $|\Delta x-\Delta y| \phi$ \\
3 & $\phi$ & $|a-b|^{2} \phi$ & $|a-\beta| \phi$ & $\sqrt{\left|x^{2}-y^{2}\right| \phi}$ \\
4 & $|a-b|^{2} \phi$ & $\phi$ & $\mu \phi$ & $|\Delta x-\Delta y| \phi$ \\
5 & $\sqrt{a b} \phi$ & $\sqrt{a b} \phi$ & $\sqrt{\mu} \phi$ & $|a \beta-\gamma| \phi$ \\
\hline
\end{tabular}

energy of rock acoustic emission. The parameter $\phi$ of Table 2 represents the maximum stress in the rock.

Under the environment and condition of rock evolution, we put forward an embedded acoustic emission characteristic analysis model. In the rock acoustic emission range, the outside world exerts many kinds of pressure. The release direction of these pressures is different. It is assumed that the rock components of different shapes are the same, according to the formula (7), the strength characteristics of rock can be analyzed.

$$
\left\{\begin{aligned}
\sigma_{x} & =\frac{h(t)}{1-\mu} \\
\sigma_{y} & =\mu g Z_{\mu}(t) \\
S_{t} & =S_{0}+\int_{0}^{t} \sigma_{x} h(t) d t \int_{0}^{t} \frac{\sigma_{y} d \mu}{d t}
\end{aligned}\right.
$$

Here, $S_{t}$ represents the strength of rock, $S_{0}$ indicates the initial rock strength. Function $h$ indicates the conversion of rock stress field. The stress intensity of the rock acoustic emission forward extrusion is indicated by $\sigma_{x}$. $\sigma_{y}$ indicates that the rock is under the earth's gravitational pull. The rock splitting rate can be calculated by the formula (8).

$$
\left\{\begin{array}{l}
v_{\text {division }}=\frac{1}{E}\left[d_{x} \cos \alpha \sum_{i=1}^{N} S_{t}+d_{y} \sin \beta\right] \\
d_{x}=\frac{\partial \sum_{x} h(t)}{\mu \partial t} \\
d_{y}=\frac{1}{\mu} \sum_{x} h(t)
\end{array}\right.
$$

\section{Performance evaluation}

A cylinder rock was used as the research object. The cylinder is $0.2-\mathrm{m}$ high, with a maximum radius of $0.15 \mathrm{~m}$. The rock is squeezed in a closed metal wall container by five cylinders. Each rock is embedded in a sensor. By extruding the metal wall container, the rock is deformed to transmit sound waves. At the bottom of the metal wall container as the origin. Sound wave propagation speed is $2000 \mathrm{~m}$ per second. The rupture point coordinates are $(0.05,0.09)$ and $(0.17,0.10)$. The second section of the establishment of the positioning algorithm (RCL) and square method location algorithm least (LSL) for acoustic emission positioning accuracy test.

We conducted a ten positioning algorithm test. The results were shown in Fig. 5. The positioning error of the proposed algorithm is less than $0.005 \mathrm{M}$. The least error of the least square method is larger than the maximum error of the proposed algorithm. Because the proposed algorithm can accurately obtain the position of acoustic emission source, combined with the embedded

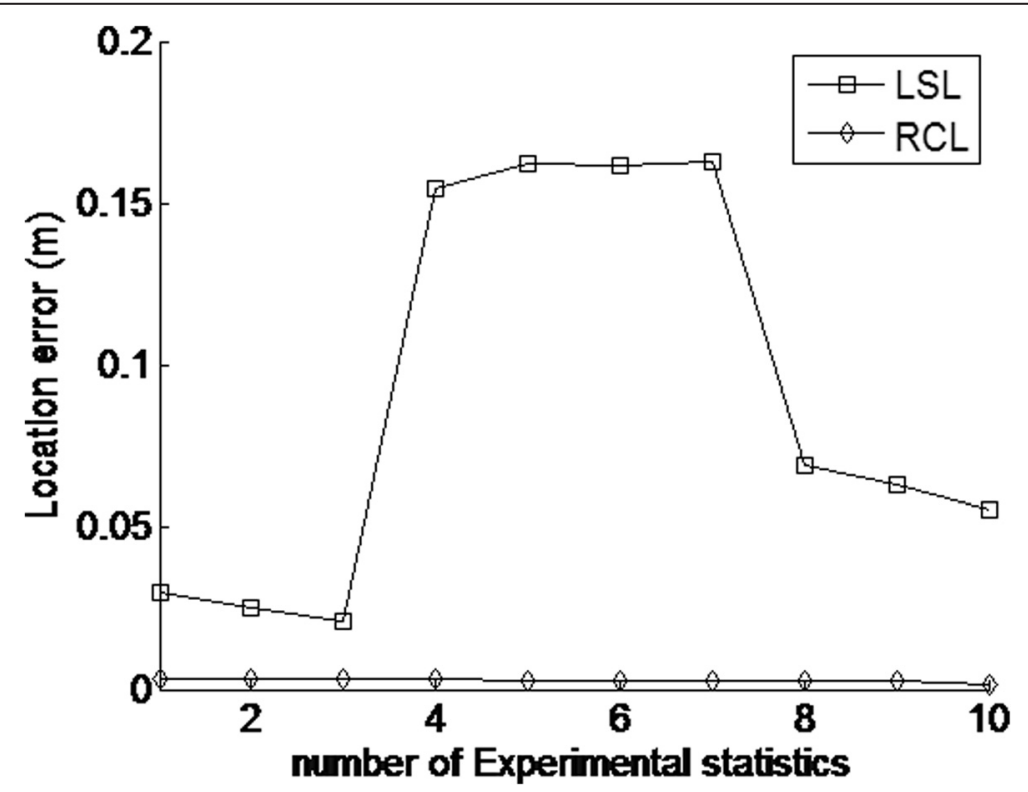

Fig. 5 Location error 


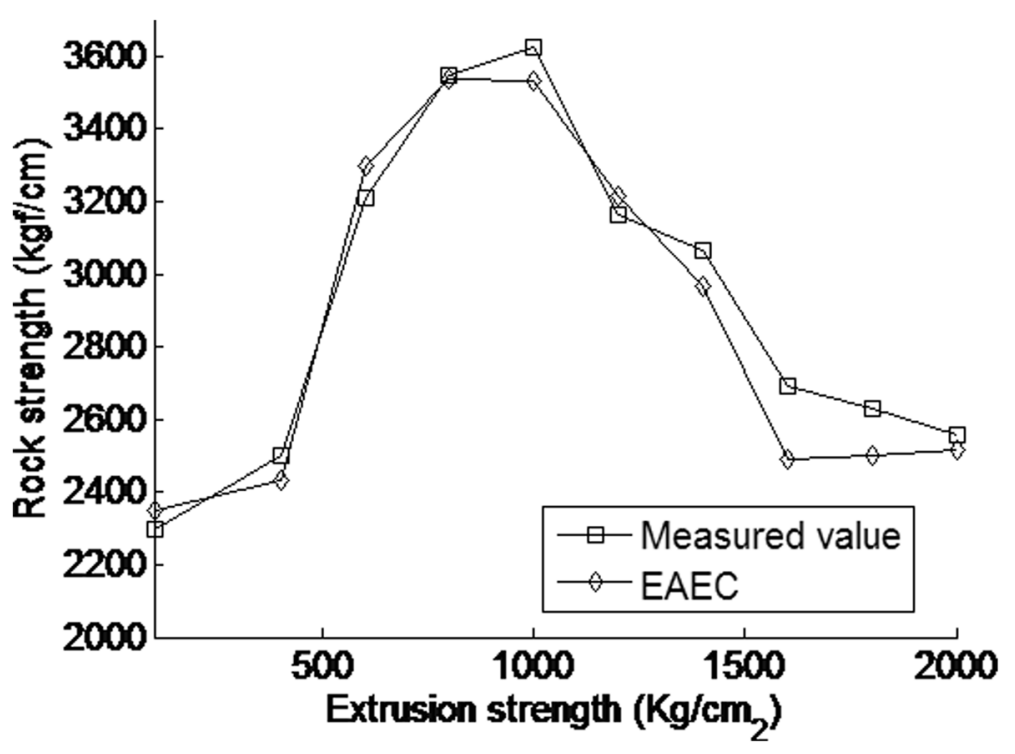

Fig. 6 Rock strength

sensor coordinates and acoustic wave propagation speed. Based on the deformation of the rock group, the moving distance is generated, and the 3D spatial simulation data is generated. The formula (4) can get the rock acoustic energy $A_{U}$. Mapping all the group wise perception points to the forward space of $Y$. Then, the $y$ to the space of the crowd sensing point is connected to the 1 closed loop to achieve the sound source localization.

In order to validate the proposed embedded characteristic analysis of acoustic emission signals (EAEC), mechanism embedded acoustic emission characteristic analysis for performance, we measured the rock group, the rock strength values of rocks, and the division rate. Then, the rock strength value, absolute energy and the rock splitting rate are calculated by using the embedded acoustic emission characteristic analysis mechanism. The comparison results are shown in Figs. 6, 7, and 8.

As can be seen from Fig. 6, as the strength extrusion becomes larger, the strength of the rock increases continuously. When the extrusion strength exceeds $1500 \mathrm{Kg} / \mathrm{cm}^{2}$, the rock strength decrease. Figure 7 shows that the EAEC can accurately capture the rock splitting rate. Figure 8 shows

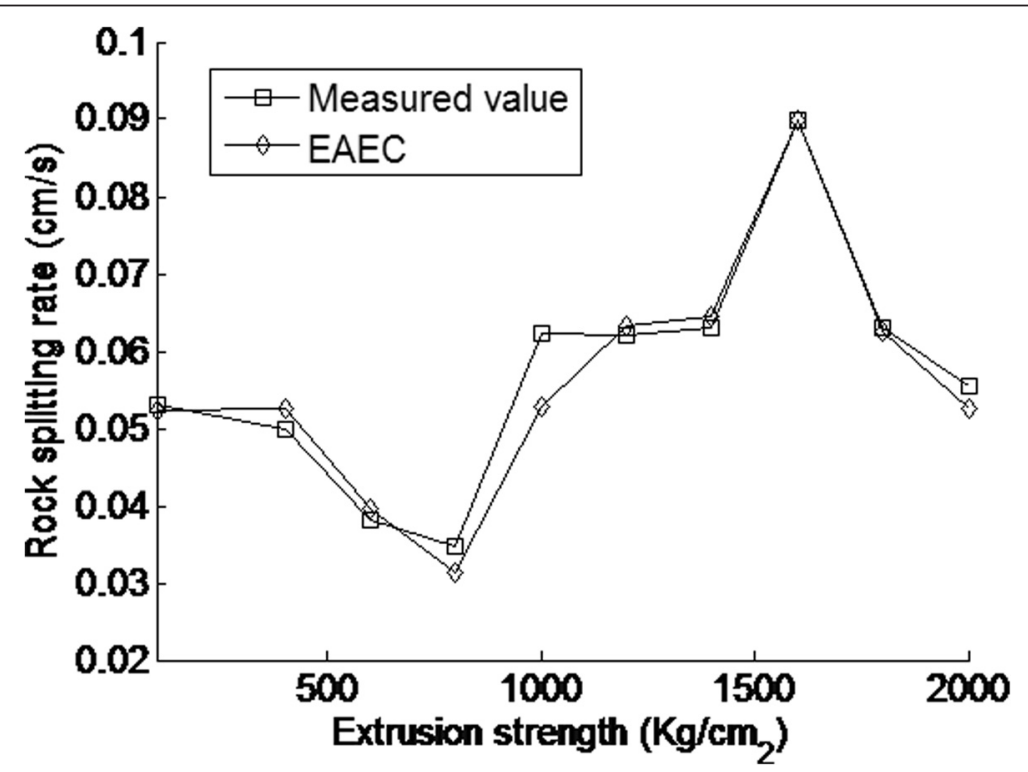

Fig. 7 Rock splitting rate 


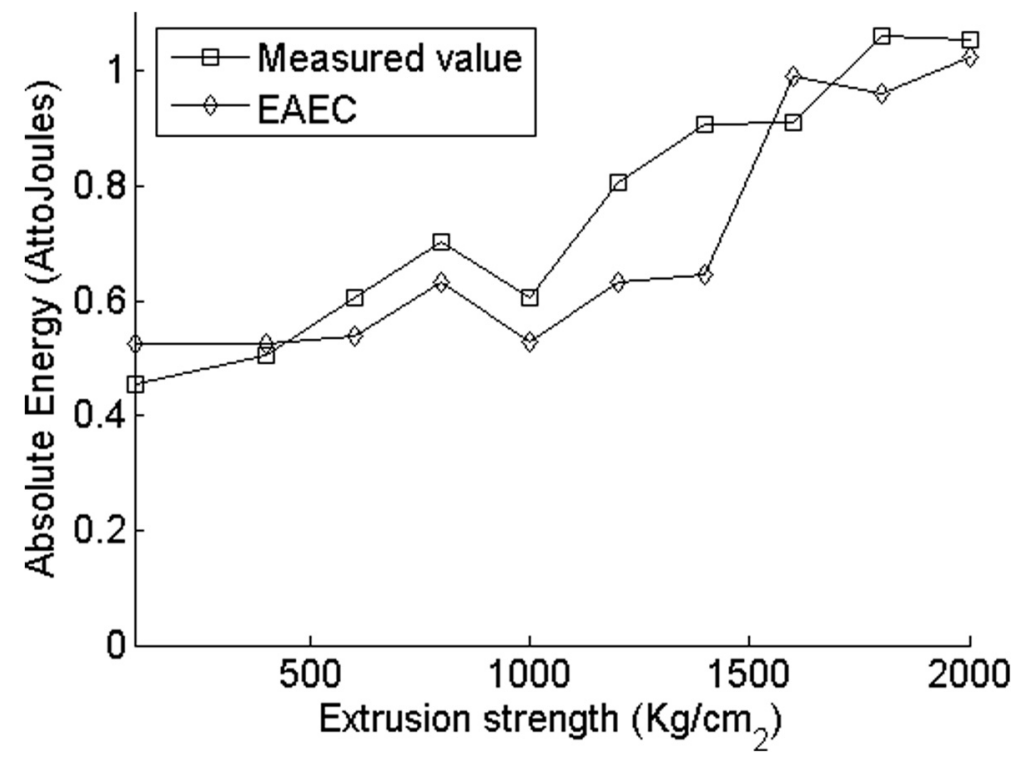

Fig. 8 Absolute energy

that the EAEC can obtain the accurate absolute energy, which is close to the measured value. This phenomenon indicates that the rock began to split. In the time dimension, the EAEC statistics of the rock sound of $N$ embedded crowd sensor nodes are monitored. EAEC obtained rock acoustic emission energy. Then, the displacement of EAEC to the fission of the rock evolution is analyzed. EAEC gets the coordinates of all the separation body. EAEC based on the acoustic emission angle and displacement distance to get the evolution factor vector of the internal structure of rock.

\section{Conclusions}

Rock acoustic emission has the advantage of nondestructive testing. It is often used to study the evolution of rocks. The cause of rock internal damage can be monitored continuously and in real time by sensing rock acoustic wave. Based on the rock sound source group intelligence localization algorithm, we propose an embedded acoustic emission characteristic analysis mechanism. The localization algorithm establishes a linear relationship between the acoustic emission and the propagation process of the rock. This algorithm can accurately restore the internal deformation and failure process of the rock. By combining the acoustic source and rock sound, we design a method of acoustic emission characteristic analysis based on 3D space. The test results show that the positioning error of the proposed algorithm is less than $0.005 \mathrm{~m}$, which is obviously better than the least square method. The calculation of rock strength and splitting rate is consistent with the actual measurement by using the embedded acoustic emission characteristic analysis mechanism.
Acknowledgements

This work is supported in part by the "Twelfth Five-year Plan" Science and Technology Research Project of The Education Department of Jilin Province, [2015] No. 281 Design of Multi-channel Rock Acoustic Emission System.

\section{Competing interest}

The author declares that he has no competing interests.

Received: 29 May 2016 Accepted: 3 August 2016

Published online: 11 August 2016

\section{References}

1. AJ Pickwell, RA Dorey, D Mba, Thick-film acoustic emission sensors for use in structurally integrated condition-monitoring applications. IEEE Transactions On Ultrasonics Ferroelectrics \& Frequency Control 58(9), 1994-2000 (2011)

2. L Jin, Q Yang, S Liu et al., Enhanced acoustic emission detection induced by electromagnetic stimulation with external magnetic field. Electromagnetic Field Computation 47(10), 3284-3287 (2011)

3. J Johnson, K Kim, S Zhang et al., High-temperature acoustic emission sensing tests using a Yttrium calcium oxyborate sensor. IEEE Transactions on Ultrasonics Ferroelectrics \& Frequency Control 61(5), 805-814 (2014)

4. P Rohwetter, W Habel, G Heidmann et al., Acoustic emission from DC pretreeing discharge processes in silicone elastomer. IEEE Transactions on Dielectrics \& Electrical Insulation 22(1), 52-64 (2015)

5. Z Cai, S Liu, C Zhang et al., Microscopic mechanism and experiment research of electromagnetically induced acoustic emission. IEEE Transactions on Magnetics 51(11), 1 (2015)

6. E Ramasso, V Placet, ML Boubakar, Unsupervised consensus clustering of acoustic emission time-series for robust damage sequence estimation in composites. IEEE Transactions on Instrumentation \& Measurement 64(12), 3297-3307 (2015)

7. $A B$ Ming, W Zhang, ZY Qin et al., Dual-impulse response model for the acoustic emission produced by a spall and the size evaluation in rolling element bearings. IEEE Transactions on Industrial Electronics 62(10), 66066615 (2015)

8. M Conti, J Willemsen, B Crispo, Providing source location privacy in wireless sensor networks: a survey. IEEE Communications Surveys \& Tutorials 15(3), 1238-1280 (2013)

9. W Tan, K Xu, D Wang, An anti-tracking source-location privacy protection protocol in WSNs based on path extension. Internet of Things Journal IEEE 1(5), 461-471 (2014)

10. Y Tang, J Hou, H Liu et al., Method for voltage sag source location based on the internal resistance sign in a single-port network. let Generation Transmission Distribution 10(7), 1720-1727 (2016) 